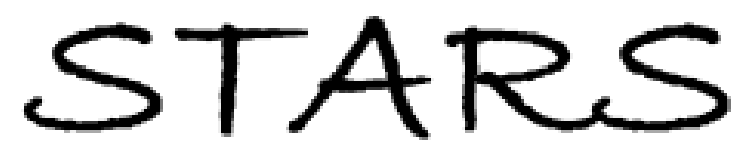

University of Central Florida

STARS

$1-1-2010$

\title{
Temperature dependence of plasmonic terahertz absorption in grating-gate gallium-nitride transistor structures
}
A. V. Muravjov
D. B. Veksler
V. V. Popov
O. V. Polischuk
N. Pala

See next page for additional authors

Find similar works at: https://stars.library.ucf.edu/facultybib2010

University of Central Florida Libraries http://library.ucf.edu

This Article is brought to you for free and open access by the Faculty Bibliography at STARS. It has been accepted for inclusion in Faculty Bibliography 2010 s by an authorized administrator of STARS. For more information, please contactSTARS@ucf.edu.

\section{Recommended Citation}

Muravjov, A. V.; Veksler, D. B.; Popov, V. V.; Polischuk, O. V.; Pala, N.; Hu, X.; Gaska, R.; Saxena, H.; Peale, R. E.; and Shur, M. S., "Temperature dependence of plasmonic terahertz absorption in grating-gate galliumnitride transistor structures" (2010). Faculty Bibliography 2010s. 570.

https://stars.library.ucf.edu/facultybib2010/570

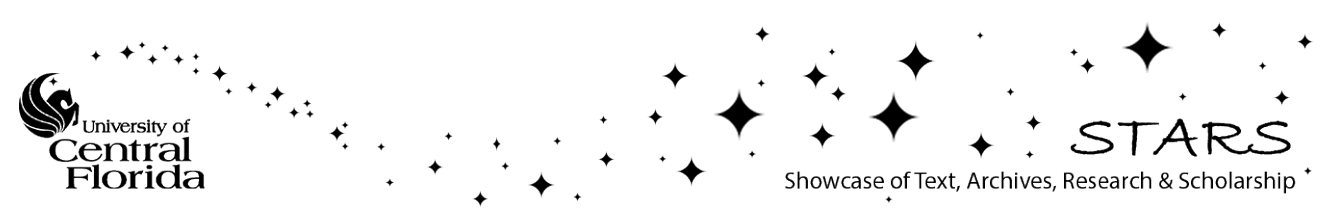




\section{Authors}

A. V. Muravjov, D. B. Veksler, V. V. Popov, O. V. Polischuk, N. Pala, X. Hu, R. Gaska, H. Saxena, R. E. Peale, and M. S. Shur 


\section{Temperature dependence of plasmonic terahertz absorption in grating-gate gallium-nitride transistor structures}

Cite as: Appl. Phys. Lett. 96, 042105 (2010); https://doi.org/10.1063/1.3292019

Submitted: 12 July 2009 . Accepted: 21 December 2009 . Published Online: 26 January 2010

A. V. Muravjov, D. B. Veksler, V. V. Popov, O. V. Polischuk, N. Pala, X. Hu, R. Gaska, H. Saxena, R. E. Peale, and M. S. Shur

\section{ARTICLES YOU MAY BE INTERESTED IN}

Terahertz amplification in RTD-gated HEMTs with a grating-gate wave coupling topology Applied Physics Letters 109, 06311 (2016); https://doi.org/10.1063/1.4961053

Electrically driven terahertz radiation of 2DEG plasmons in AIGaN/GaN structures at $110 \mathrm{~K}$ temperature

Applied Physics Letters 110, 202101 (2017); https://doi.org/10.1063/1.4983286

Rational design of high-responsivity detectors of terahertz radiation based on distributed self-mixing in silicon field-effect transistors

Journal of Applied Physics 105, 114511 (2009); https://doi.org/10.1063/1.3140611

\section{Applied Physics Reviews} Now accepting original research 


\title{
Temperature dependence of plasmonic terahertz absorption in grating-gate gallium-nitride transistor structures
}

\author{
A. V. Muravjov, ${ }^{1,2, a)}$ D. B. Veksler, ${ }^{1}$ V. V. Popov ${ }^{3}$ O. V. Polischuk, ${ }^{3}$ N. Pala, ${ }^{4}$ X. Hu, ${ }^{5}$ \\ R. Gaska, ${ }^{5}$ H. Saxena, ${ }^{6}$ R. E. Peale, ${ }^{6}$ and M. S. Shur ${ }^{1}$ \\ ${ }^{1}$ Department of Electrical, Computer, and Systems Engineering, Rensselaer Polytechnic Institute, Troy, New \\ York 12180, USA \\ ${ }^{2}$ Institute for Physics of Microstructures, RAS, Nizhny, Novgorod 603950, Russia \\ ${ }^{3}$ Kotelnikov Institute of Radio Engineering and Electronics, RAS, Saratov 410019, Russia \\ ${ }^{4}$ Electrical and Computer Engineering, Florida International University, Miami, Florida 33174, USA \\ ${ }^{5}$ Sensor Electronic Technology, Inc., 1195 Atlas Road, Columbia, South Carolina 29209, USA \\ ${ }^{6}$ Department of Physics, University of Central Florida, Orlando, Florida 32816, USA
}

(Received 12 July 2009; accepted 21 December 2009; published online 26 January 2010)

\begin{abstract}
Strong plasmon resonances have been observed in the terahertz transmission spectra $(1-5 \mathrm{THz})$ of large-area slit-grating-gate $\mathrm{AlGaN} / \mathrm{GaN}$-based high-electron-mobility transistor (HEMT) structures at temperatures from 10 to $170 \mathrm{~K}$. The resonance frequencies correspond to the excitation of plasmons with wave vectors equal to the reciprocal lattice vectors of the metal grating, which serves both as a gate electrode for the HEMT and a coupler between plasmons and incident terahertz radiation. Wide tunability of the resonances by the applied gate voltage demonstrates potential of these devices for terahertz applications. (C) 2010 American Institute of Physics.
\end{abstract}

[doi:10.1063/1.3292019]

Plasma excitations (plasmons) in field-effect transistors with two-dimensional (2D) electron channels strongly affect terahertz (THz) properties of these devices. ${ }^{1}$ Faster plasmon velocities in comparison with electron drift velocities enable plasmonic devices to break into the $\mathrm{THz}$ range of operation frequencies. ${ }^{1-11}$ Critical issues for future development of the plasmon electronics are ensuring a strong coupling between plasmons and $\mathrm{THz}$ radiation and feasibility of room temperature operation. Previously, pronounced 2D plasmons in $\mathrm{Si}^{2,3}$ III-V compound, ${ }^{5-12}$ and $\mathrm{GaN}^{13}$ based semiconductor structures were observed only below liquid nitrogen temperatures.

In this letter, we present our results on the observation of plasmon resonances in large-area slit-grating-gate $\mathrm{AlGaN} /$ GaN-based high-electron-mobility transistor (HEMT) structures (Fig. 1) at temperatures up to $170 \mathrm{~K}$ in agreement with theoretical predictions of Ref. 14. The AlGaN/GaN heterostructure was grown on sapphire substrate by migration enhanced metal-organic chemical vapor deposition $\left(\right.$ MEMOCVD $\left.^{\circledR}\right)$ technique. The heterostructure consisted of a $100 \mathrm{~nm}$ thick AlN buffer layer, $1.4 \mu \mathrm{m}$ thick undoped GaN layer, followed by $\mathrm{Al}_{0.2} \mathrm{Ga}_{0.8} \mathrm{~N}$ barrier layer, Si-doped to approximately $2 \times 10^{18} \mathrm{~cm}^{-3}$. A metal grating gate with the period $L=1.5 \mu \mathrm{m}$ was deposited on top of the structure. Individual gate-finger length was $W=1.15 \mu \mathrm{m}$ with $0.35 \mu \mathrm{m}$ slits between adjacent fingers. The thickness of the AlGaN barrier layer was $d=28 \pm 2 \mathrm{~nm}$. Contacts for the source/drain and gate electrodes were fabricated using e-beam evaporated $\mathrm{Ti} / \mathrm{Al} / \mathrm{Ti} / \mathrm{Au}$ and $\mathrm{Ni} / \mathrm{Au}$ metal stacks, respectively. The metal grating served both as a gate electrode and a coupler between plasmons and incident $\mathrm{THz}$ radiation. The 2D electron concentration $N_{1}$ in the channel under the grating-gate fingers was controlled by the gate voltage $U_{\mathrm{g}}$ referenced to the connected source and drain electrodes. The electron sheet density in the channel under grating-gate fin-

${ }^{a)}$ Electronic mail: amuravie@gmail.com. gers can be estimated as $N_{1}=\varepsilon \varepsilon_{0}\left(U_{\mathrm{g}}-U_{\text {th }}\right) / e d$, where $U_{\text {th }}$ is the channel depletion threshold voltage, $\varepsilon$ is the dielectric constant of the barrier layer, $\varepsilon_{0}$ is the free-space permittivity, and $e$ is the electron charge. The threshold voltage estimated from the transconductance measurements is $U_{\mathrm{th}} \approx-4 \mathrm{~V}$, which yields $N_{1} \approx 7.5 \times 10^{12} \mathrm{~cm}^{-2}$ for $U_{\mathrm{g}}=0$ at room temperature. Large active area of the device $\left(1.6 \times 1.7 \mathrm{~mm}^{2}\right.$ with more than 1000 metal fingers) allowed efficient focusing of incident $\mathrm{THz}$ radiation and its study by methods of conventional Fourier transform infrared (FTIR) spectroscopy.

Transmission spectra of the structure were measured by Bruker IFS $66 \mathrm{v} / \mathrm{S}$ FTIR spectrometer at liquid nitrogen temperatures and above, ${ }^{15,16}$ and by Bomem DA8 Fourier spectrometer below liquid nitrogen temperature (see Ref. 12 for details). In both experiments, the original spectral resolution was $0.03 \mathrm{THz}$, which was sufficient for resolving the FabryPerot fringes $(\Delta \nu=0.1 \mathrm{THz})$ caused by multiple reflections between the structure and the back of the sapphire substrate. To eliminate these interference effects, the spectra were averaged over the period of the Fabry-Perot oscillations, which reduced the effective resolution to $0.1 \mathrm{THz}$. The transmission

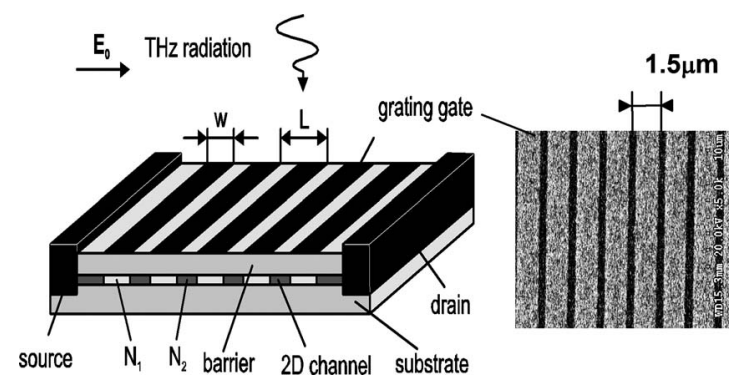

FIG. 1. Grating-gate HEMT structure (left). THz radiation with polarization of the electric field across the gate fingers is incident from the top; scanning electron microscopy image of the grating-gate fragment with $L=1.5 \mu \mathrm{m}$, $W=1.15 \mu \mathrm{m}$ (right). 

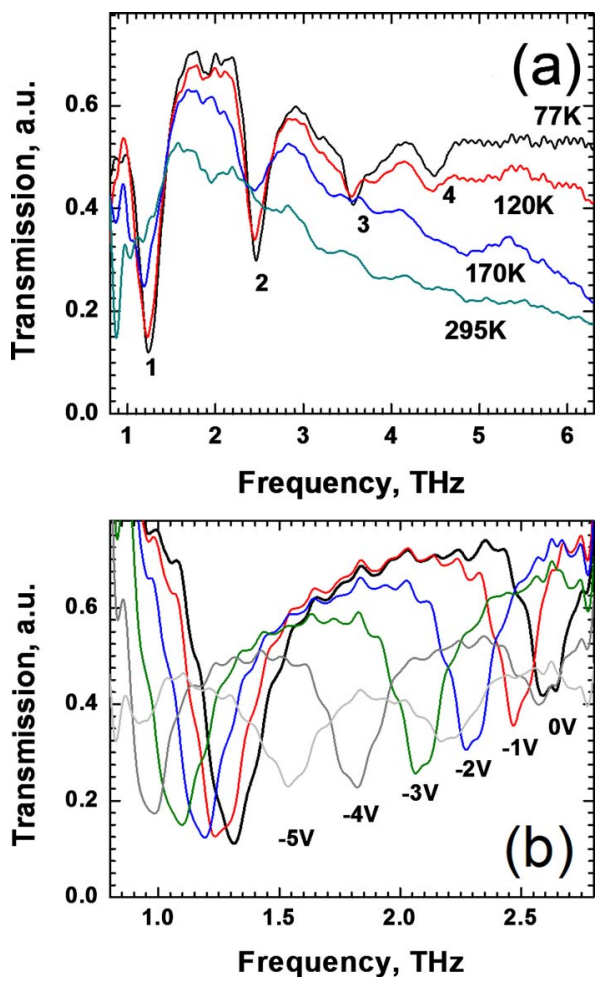

FIG. 2. (Color online) (a) Transmission spectra of the grating-gate AlGaN/ GaN HEMT structure, referenced to free space, measured at temperatures from $77 \mathrm{~K}$ to $295 \mathrm{~K}$ for $U_{\mathrm{g}}=0$. Resolution is $0.1 \mathrm{THz}$. (b) Transmission spectra for different applied gate voltages $U_{\mathrm{g} \text {,appl }}=(0 \mathrm{~V} ;-1 \mathrm{~V} ;-2 \mathrm{~V}$; $-3 \mathrm{~V} ;-4 \mathrm{~V}$; and $-5 \mathrm{~V}$ ) at $T=10 \mathrm{~K}$. Resolution is $0.1 \mathrm{THz}$. The spectra are referenced to the transmission spectrum of the identical $A 1 G a N / G a N$ device without metal gate grating.

spectra measured at temperatures 77,120 , and $170 \mathrm{~K}$ exhibit well pronounced resonances at the fundamental plasma frequency and its second, third, and fourth harmonics [Fig. $2(\mathrm{a})]$. Observed resonance frequencies are in a good agreement with plasmon dispersion law $\omega_{\mathrm{p}}=s k_{\mathrm{p}}$ and selection rule for the plasmon wave vector as $k_{\mathrm{p}}=2 \pi n / L$ $(n=1,2,3,4 \ldots){ }^{17}$ where the plasmon velocity is $s$ $=\left(e^{2} N_{1} d / m^{*} \varepsilon \varepsilon_{0}\right)^{1 / 2}$ with $m^{*}$ being the electron effective mass. For $N_{1}=7.5 \times 10^{12} \mathrm{~cm}^{-2}$, one obtains $s \approx 1.9$ $\times 10^{8} \mathrm{~cm} / \mathrm{s}$. The resonances obey this simple dispersion law for gated plasmons due to relatively large grating-gate duty cycle $W / L \approx 0.77$ and small gate-to-channel separation $d$ $\ll L$. However, because of alternating gated and ungated regions, the actual value of $s$ is also a weak function of the ratio $W / L$ and of the resonance order $n$, which results in slightly nonequidistant behavior of the resonances exhibited in Fig. 2(a). Figure 2(b) demonstrates tunability of the resonances by the applied gate voltage $U_{\mathrm{g}}$. Negative $U_{\mathrm{g}}$ decreases the electron concentration $N_{1}$ under the gate fingers, decreasing plasmon velocity $s$ and, hence, causing redshift of the resonances. The best fit of the resonance shift versus $U_{\mathrm{g}}$ for the first, second, and third resonances in Fig. 2(b) corresponds to $U_{\mathrm{th}}^{*} \approx-8 \mathrm{~V}$, while the value of $U_{\mathrm{th}}$ found from the transconductance measurements is about $-4 \mathrm{~V}$. We explain this by a voltage drop across the channel caused by the gate leakage current crowding at the edges of the structure. The gate leakage conductivity is about $0.005 \Omega^{-1}$ at $T=10 \mathrm{~K}$, while the conductivity of the channel is about $0.02 \Omega^{-1}$ (for $\left.U_{\mathrm{g}}=0\right)$. As a result, the applied gate voltage $U_{\mathrm{g}, \text { appl }}$ [specified in Fig. 2(b)] appears almost two times higher than the actual
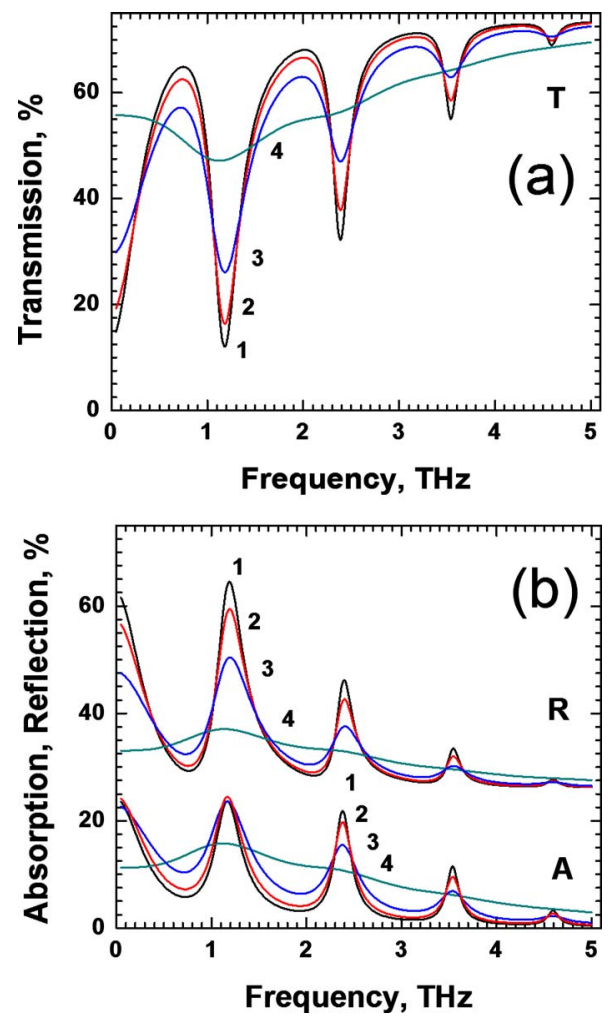

FIG. 3. (Color online) (a) Calculated transmission $(T)$ spectra of the gratinggate $\mathrm{AlGaN} / \mathrm{GaN}$ HEMT structure for different electron mobilities: (1) $\mu_{77 \mathrm{~K}}=10000 \mathrm{~cm}^{2} / \mathrm{V} \mathrm{s}\left(\tau=1.14 \times 10^{-12} \mathrm{sec}\right)$, (2) $\mu_{120 \mathrm{~K}}=7600 \mathrm{~cm}^{2} / \mathrm{V} \mathrm{s}$ $\left(\tau=0.86 \times 10^{-12} \mathrm{sec}\right),(3) \mu_{170 \mathrm{~K}}=4400 \mathrm{~cm}^{2} / \mathrm{V} \mathrm{s}\left(\tau=0.5 \times 10^{-12} \mathrm{sec}\right)$, and (4) $\mu_{295 \mathrm{~K}}=1200 \mathrm{~cm}^{2} / \mathrm{V} \mathrm{s}\left(\tau=0.14 \times 10^{-12} \mathrm{sec}\right)$. (b) Corresponding calculated absorption $(A)$ and reflection $(R)$ spectra.

voltage $U_{\mathrm{g}}$ between the gates and the channel in the central part of the structure.

Numerical simulations of the $\mathrm{THz}$ plasmon transmission spectra were performed using the approach developed in Ref. 18. The response of $2 \mathrm{D}$ electrons to incident $\mathrm{THz}$ radiation was described by the sheet conductivity using the local Drude model as

$$
\sigma(\omega)=\frac{e^{2} N_{1,2} \tau}{m^{*}(1-i \omega \tau)},
$$

where $N_{1}$ and $N_{2}$ are the steady-state electron densities in the gated and ungated parts of the channel, respectively (see Fig. 1 ), and $\tau$ is a phenomenological electron scattering time related to the electron mobility, $\mu$, as $\tau=\mu \mathrm{m}^{*} / e$. Parameters of the structure used in the numerical simulations were $m^{*}$ $=0.2 m_{0}$ with $m_{0}$ being the free-electron mass, $\varepsilon=9.5, d$ $=28 \mathrm{~nm}, N_{1}=N_{2}=7.5 \times 10^{12} \mathrm{~cm}^{-2}$. Gold fingers of the grating gate were considered as $2 \mathrm{D}$ strips with the sheet conductivity equal to $2.5 \mathrm{~S}$. However the assumption of perfectly conductive strips yielded no significant difference in the results. The electron mobility $\mu$ was used as a fitting parameter in the simulations in order to achieve best correspondence between linewidths and intensities of the plasmon resonances in the calculated (Fig. 3) and measured [Fig. 2(a)] transmission spectra for each temperature. These fitted values of $\mu$ (see the caption of Fig. 3) are in good agreement with previously reported electron mobilities in $\mathrm{AlGaN} / \mathrm{GaN}$ heterostructures at the same temperatures. ${ }^{19}$

Due to relatively high electron concentration in $\mathrm{AlGaN} /$ $\mathrm{GaN}$ heterojunction, the criterion for resolving adjacent reso- 
nance lines $\left(\omega_{\mathrm{p}} \tau_{\mathrm{p}} \gg 1\right)$ is satisfied up to $170 \mathrm{~K}$. Plasmon relaxation time $\tau_{\mathrm{p}}$ in our numerical simulations was defined by the electron scattering rate and plasmon radiative decay. ${ }^{20}$ Accurate comparison between the experimental and calculated linewidths of the resonances allowed us to evaluate the role of electron fluid viscosity $\nu$, as another plasmon damping mechanism ${ }^{4}$ potentially affecting $\tau_{\mathrm{p}}$. Viscosity contribution to the resonance linewidth is $2 \nu k_{\mathrm{p}}^{2}$, and, hence, should be especially pronounced for plasmon modes with larger wave vectors. ${ }^{4}$ Slight broadening of the higher-order resonances in the measured spectra beyond the numerically simulated linewidths allowed us to estimate the upper bound for the electron viscosity as $10 \mathrm{~cm}^{2} / \mathrm{s}$.

In conclusion, the observed depth of the resonances is the result of strong coupling between incident $\mathrm{THz}$ radiation and plasmons (high plasmon radiative decay rate), ${ }^{20}$ achieved by using a grating gate with relatively narrow slits. ${ }^{14}$ At elevated temperatures faster plasmon radiative decay, and thus narrower slits, are required to satisfy the conditions of optimal coupling, where the absorption of the radiation by plasmons reaches a maximum $A=0.5\left(1-\sqrt{R_{0}}\right)$ ( $R_{0} \approx 0.25$ is the reflectivity of sapphire), and the plasmon radiative decay rate is equal to $1 / 2$ of the electron scattering rate. ${ }^{20}$ In our case, such conditions are achieved at the temperature $120 \mathrm{~K}$ [Fig. 3(b)]. Below $120 \mathrm{~K}$ the radiative decay of plasmons becomes dominant causing efficient resonant reflection from the plasma layer with $R$ reaching $65 \%$ at $77 \mathrm{~K}$.

The work at RPI was supported by the U.S. ONR. The work at SET has been partially supported by NASA STTR Phase I under Contract No. NNX09CF26P (Program Monitor Dr. Nurul Abedin). The work at the Kotelnikov Institute was supported by the RFBR (Grant Nos. 09-02-00395 and 08-02-92497) and by the Russian Academy of Sciences Program "Fundamentals of Nanotechnologies and Nanomaterials." A.V.M. thanks RFBR for partial support of work at IPM RAS (Grant No. 07-02-01487a). R.E.P. and H.S. thank Air Force Research Laboratory, Hanscom AFB MA for supporting the work at UCF (Grant No. FA871807C0036).
${ }^{1}$ M. S. Shur and J.-Q. Lü, IEEE Trans. Microwave Theory Tech. 48, 750 (2000).

${ }^{2}$ S. J. Allen, D. C. Tsui, and R. A. Logan, Phys. Rev. Lett. 38, 980 (1977).

${ }^{3}$ D. C. Tsui, E. Gornik, and R. A. Logan, Solid State Commun. 35, 875 (1980).

${ }^{4}$ M. Dyakonov and M. S. Shur, Phys. Rev. Lett. 71, 2465 (1993).

${ }^{5}$ X. G. Peralta, S. J. Allen, M. C. Wanke, N. E. Harff, J. A. Simmons, M. P. Lilly, J. L. Reno, P. J. Burke, and J. P. Eisenstein, Appl. Phys. Lett. 81, 1627 (2002).

${ }^{6}$ E. A. Shaner, M. Lee, M. C. Wanke, A. D. Grine, J. L. Reno, and S. J. Allen, Appl. Phys. Lett. 87, 193507 (2005).

${ }^{7}$ F. Teppe, D. Veksler, A. P. Dmitriev, V. Yu. Kachorovskii, S. Rumyantsev, W. Knap, and M. S. Shur, Appl. Phys. Lett. 87, 052107 (2005).

${ }^{8}$ W. J. Stillman and M. S. Shur, J. Nanoelectron. Optoelectron. 2, 209 (2007).

${ }^{9}$ A. El Fatimy, F. Teppe, N. Dyakonova, W. Knap, D. Seliuta, G. Valušis, A. Shchepetov, Y. Roelens, S. Bollaert, A. Cappy, and S. Rumyantsev, Appl. Phys. Lett. 89, 131926 (2006).

${ }^{10}$ A. Shchepetov, C. Gardès, Y. Roelens, A. Cappy, S. Bollaert, S. Boubanga-Tombet, F. Teppe, D. Coquillat, S. Nadar, N. Dyakonova, H. Videlier, W. Knap, D. Seliuta, R. Vadoclis, and G. Valušis, Appl. Phys. Lett. 92, 242105 (2008).

${ }^{11}$ S. Boubanga-Tombet, F. Teppe, D. Coquillat, S. Nadar, N. Dyakonova, H. Videlier, W. Knap, A. Shchepetov, C. Gardès, Y. Roelens, S. Bollaert, D. Seliuta, R. Vadoclis, and G. Valušis, Appl. Phys. Lett. 92, 212101 (2008).

${ }^{12}$ H. Saxena, R. E. Peale, and W. R. Buchwald, J. Appl. Phys. 105, 113101 (2009).

${ }^{13}$ A. El Fatimy, S. Boubanga Tombet, F. Teppe, W. Knap, D. B. Veksler, S. Rumyantsev, M. S. Shur, N. Pala, R. Gaska, Q. Fareed, X. Hu, D. Seliuta, G. Valusis, C. Gaquiere, D. Theron, and A. Cappy, Electron. Lett. 42, 1342 (2006).

${ }^{14}$ V. V. Popov, G. M. Tsymbalov, D. V. Fateev, and M. S. Shur, Int. J. High Speed Electron. Syst. 17, 557 (2007).

${ }^{15}$ A. V. Muravjov, D. B. Veksler, V. V. Popov, M. S. Shur, N. Pala, X. Hu, R. Gaska, H. Saxena, and R. E. Peale, International Conference on Optical Terahertz Science and Technology, Santa Barbara, CA, 7-11 March 2009, MA5 (unpublished).

${ }^{16}$ A. V. Muravjov, D. B. Veksler, X. Hu, R. Gaska, N. Pala, H. Saxena, R. E. Peale, and M. S. Shur, Proc. SPIE 7311, 73110D (2009).

${ }^{17}$ L. Zheng, W. L. Schaich, and A. H. MacDonald, Phys. Rev. B 41, 8493 (1990)

${ }^{18}$ V. V. Popov, G. M. Tsymbalov, and M. S. Shur, J. Phys.: Condens. Matter 20, 384208 (2008).

${ }^{19}$ R. Gaska, J. W. Yang, A. Osinsky, Q. Chen, M. Asif Khan, A. O. Orlov, G. L. Snider, and M. S. Shur, Appl. Phys. Lett. 72, 707 (1998).

${ }^{20}$ V. Popov, O. V. Polischuk, T. V. Teperik, X. G. Peralta, S. J. Allen, N. J. M. Horing, and M. C. Wanke, J. Appl. Phys. 94, 3556 (2003). 